\title{
Diagnóstico de la calidad de vida laboral, engagement y burnout en trabajadores de una institución de educación superior del Ecuador
}

\section{Diagnosis of quality of work life, engagement and burnout in workers of a higher education institution of Ecuador}

\author{
María Isabel Armijo Mora \\ Universidad de Especialidades Espíritu Santo, Ecuador \\ Cecilia Alexandra Portalanza Chavarría \\ Universidad de Especialidades Espíritu Santo, Ecuador
}

Autor para correspondencia: marmijo@uees.edu.ec, aportalanza@uees.edu.ec Fecha de recepción: 16 de junio 2017 - Fecha de aceptación: 30 de noviembre de 2017

Resumen: Durante las últimas décadas en Ecuador, las Instituciones de Educación Superior (IES) se han visto envueltas en cambios de origen regulatorios que las obligan a realizar ajustes en sus estructuras afectando a sus colaboradores. El objetivo central de este artículo es estudiar la calidad de vida laboral, el engagement y el burnout en los trabajadores de las áreas administrativas y académicas de una IES. Se inicia con una revisión de la literatura de los constructos calidad de vida laboral, engagement y burnout, sus definiciones y principales características. A través de un estudio empírico realizado a 265 trabajadores de una IES se determina su nivel de calidad de vida y se identifican las dimensiones que más contribuyen a su engagement. De la misma forma, se evalúa el burnout y se presentan las variables que más inciden. A manera general se concluye que existe un buen nivel de engagement, una percepción de calidad de vida laboral alto y un bajo nivel de burnout.

Palabras clave: engagement; calidad de vida profesional; burnout

\begin{abstract}
During the last decades in Ecuador, the Institutions of Higher Education (IHE) have been involved in regulatory changes that force them to make adjustments in their structures affecting their personnel. The main objective of this article is to study the quality of working life, engagement and burnout among workers in the administrative and academic areas of an IHE. It begins with a review of the literature on the constructs of quality of work life, engagement and burnout, their definitions and main characteristics. Within an empirical study from 265 workers of an HEI that will determine their level of quality of life and identifies the dimensions that most contribute to their engagement. In the same way, the burnout is evaluated and the variables that most affect are presented. In general, it is concluded that there is a good level of engagement, a perception of a high quality of work life and a low level of burnout.
\end{abstract}

Key words: quality of work life; engagement; burnout 


\section{Introducción}

En la actualidad, las empresas buscan personas que puedan garantizarles efectividad, productividad y calidad (Suescùn-Carrero, Sarmiento, Alvarez, \& Lugo, 2016). Esta exigencia ha hecho que las organizaciones se cuestionen la necesidad de plantear nuevas formas de organizarse y establecer parámetros para que sus empleados puedan ser felices y desempeñar mejor su trabajo.

La calidad de vida laboral debe ser mantenida para asegurar que los trabajadores puedan conservar un nivel alto de engagement en su trabajo diario (Indumathy, 2012). En diversas empresas se considera que hay diferentes factores que afectan la calidad de vida laboral y hacen que por este motivo existan niveles de burnout y de engagement entre sus trabajadores (Chandranshu, 2012).

De igual manera, se ha demostrado que la calidad de vida laboral tiene efectos en el burnout (Zia, Rezvani, \& Fattaneh, 2014). Es importante identificar los posibles efectos del burnout antes que los empleados puedan llegar a tener el síndrome, y de igual manera antes que las empresas puedan verse perjudicadas por ellos.

Paris \& Omar (2008) exponen que si existiera menor índice de burnout la calidad de vida aumentaría. Así mismo Herrera-Amaya \& Manrique-Abril (2008) se enfocan en la calidad de vida laboral, donde si el engagement disminuye, llegará a burnout afectando su calidad de vida profesional.

Por otro lado, un escritor anónimo indica (2002) que los factores más influyentes sobre el burnout son la carga de trabajo, el control que tengan los empleados por parte de los superiores, los beneficios y valoración ante su trabajo entre otros.

El engagement, en una organización debe ser evidenciada a través de los resultados de la empresa (Solomon, Employee Engagement: The Key to Improving Performance, 2010). Los trabajadores que se encuentran con un alto nivel de engagement serán aquellos que perduren en la organización y puedan de igual manera crecer con ella y alcanzar una calidad de vida laboral mayor a través de satisfacción laboral (Patton, 2012).

En el sector de la educación, los docentes pueden migrar de un estado de engagement a burnout debido a cambios en su vida laboral causados por aspectos de motivación y despersonalización del trabajo (Esteras, Chorot, \& Sandin, 2014).

En el Ecuador, el sector de la educación desde el año 2010 ha venido atravesando cambios dados por la Ley Orgánica de Educación Superior, (LOES) lo que obliga a las IES adaptarse para cumplir estas nuevas disposiciones. Los trabajadores de estas instituciones son quienes deben llevar a cabo estos cambios. Son ellos los que deben asumir ya sean nuevas funciones, nuevas cargas de trabajo y más responsabilidades que pueden afectar a su calidad de vida laboral y como consecuencia disminuir su engagement y aumentar su nivel de burnout. 
De esta manera, se vuelve importante evaluar cómo la calidad de vida laboral incide en el engagement y burnout de los trabajadores de una IES.

En este estudio, se inicia con una revisión de la literatura de los constructos de calidad de vida laboral, engagement y burnout, sus definiciones, estudios previos y sus principales factores. A través de un estudio empírico aplicado a docentes tiempo completo y personal administrativo de una IES se identifican los niveles de calidad de vida laboral y se evalúa la existencia o no de la misma. Así mismo, se identificarán las dimensiones de engagement y burnout que inciden en los trabajadores.

\section{Revisión de la literatura}

A continuación, se analizan las diferentes características encontradas de la revisión literaria concerniente al burnout, engagement y calidad de vida laboral. Se mencionarán áreas referentes a la despersonalización, calidad de vida laboral, liderazgo, las relaciones interpersonales y la motivación en los puestos de trabajo. De igual manera se plantean otras temáticas de interés que están relacionadas directamente al objeto de estudio.

\section{Calidad de vida laboral}

Existen diferentes teorías en el área de la calidad de vida laboral. Nadler \& Lawler (1983) analizaron este concepto que se inició en la década de 1980 donde se busca sensibilizar el desarrollo del recurso humano y descubrir la mejor manera para obtener una calidad de vida óptima y una calidad laboral próspera.

En lo que refiere al concepto Kanfer (1990), este define la calidad de vida laboral como todas las actitudes que tienen los trabajadores en sus empleos. Este autor, trata de analizar aquellos factores que hacen que su permanencia en el lugar de trabajo sea placentera, tanto para la compañía como para los colaboradores. El determina que algunos factores que influyen son los salarios, los ascensos, las condiciones de trabajo y como éstos influyen en su día a día.

Según Segurado \& Agullo (2002) se debe buscar mayor sensibilización ante las personas que trabajan en una empresa, buscando condiciones de trabajo seguras, saludables, satisfaciendo no solamente sus necesidades sino también sus peticiones ofreciendo así oportunidades de desarrollo, para garantizar una óptima calidad de vida laboral.

De igual manera, desde la perspectiva empresarial, Senge (2006) analiza el mundo de las organizaciones y claramente señala un ganador, quien es aquella persona que aprende de manera rápida y eficaz, siendo más estructurado y se enfoca en los pequeños detalles. Todo esto ayudará al individuo a utilizar lo aprendido para mejorar su puesto de trabajo y en general su organización. Si un trabajador tiene una alta calidad de vida laboral, será trasladada en su vida personal y así se sentirá aceptado tanto en la sociedad como en su día a día y podrá encontrar un equilibrio entre su vida y su trabajo estando estable y alerta para continuar su vida diaria.

Cooper (2009), demuestra que, mejorando la calidad de vida laboral en las empresas, existe mayor rendimiento, ventas y ganancias. Este concepto es asociado a múltiples factores 
tanto internos como externos, donde el interés principal es encontrar un punto de equilibrio del trabajador con la empresa.

De igual manera, la calidad de vida laboral gira entorno de actitudes generales del individuo hacia su trabajo, y esto no sólo le afecta en su trabajo diario si no que influye así mismo en su vida personal y en sus relaciones interpersonales (Robbins, 2012).

Ardila (2003) reconoce que la calidad de vida laboral es un estado de complacencia en el individuo. No solo se busca el bienestar material, sino una satisfacción no monetaria para poder llegar a un nivel de engagement dentro de la organización y así evitar tener empleados con el síndrome de burnout.

\section{Engagement}

Kahn (1990), define el engagement como un estado de vigor, dedicación y absorción en un trabajador de una institución. De igual manera, Montoya \& Moreno (2012) indican que aquellos trabajadores con alto niveles de engagement son aquellas personas que se conectan en su trabajo, muestran interés, vitalidad, involucramiento y dedicación.

Así mismo, como lo determina Adler (2008), aquella persona que está engaged lo muestra de manera física y emocional durante sus actividades de trabajo. Igualmente, la calidad y eficacia de los trabajadores en la organización es indispensable y así mismo identificar factores para poder llegar a un nivel alto de engagement de sus empleados, esto luego se verá reflejado en la productividad y eficiencia de la organización (Solomon, 2010).

Existen dos escuelas del engagement, la primera es la que mencionan Maslach \& Leiter (1997) donde la energía, el involucramiento laboral y la eficacia son lo opuesto a las dimensiones que detalla el burnout. La otra escuela analiza el engagement como un "estado afectivo, positivo relacionado con el trabajo, el cual se caracteriza por vigor, dedicación y absorción" (Schaufeli, Salanova, Gonzalez-Roma, \& Bakker, 2002).

Las escuelas antes mencionadas iniciaron siendo un tema de rivalidad entre el trabajador y el empleador. Luego se identificó que el engagement les provee mayor calidad de vida laboral y ésta se encuentra ligada a la seguridad, salud y bienestar de los colaboradores (Houdmont, Leka, \& Bulger, 2008).

Porter, Bigley \& Steers (2002) analizan uno de los factores del engagement, siendo este el vigor, como la motivación, la fuerza que le da energía y resiliencia a los trabajadores. Sin embargo, esta motivación no solo lleva a las personas a tomar buenas o malas decisiones, sino que además influye la forma, dirección, efectividad, y duración de la conducta de cada uno de ellos (Meyer, Becker, \& Vandenberghe, 1989).

La dedicación es otro factor importante para el engagement, este se centra como lo indica VanScotter \& Motowidlo (1997), en el comportamiento para seguir reglas, trabajar arduamente, tomar iniciativa y resolver problemas en el trabajo. La dedicación es el motor principal para la 
motivación laboral y para promover los intereses de la organización con un sentimiento de orgullo e inspiración.

La absorción, para Shaufeli, Salanov, Gonzalez-Roma \& Bakker (2002), está caracterizada por estar completamente concentrada y comprometida en el trabajo. Otra característica de la absorción es que el trabajador no se da cuenta del tiempo transcurrido y muchas veces tiene dificultades para separarse de sus labores una vez terminada la jornada (Jenaro, Flores, Begoña, \& Cruz, 2010).

Ocampos, Juarez, Arias \& Hindrichs (2015), identifican que aquellos trabajadores que cuentan con los factores del engagement son aquellos que suman a una organización y pueden llegar a cumplir muchos años en la institución y un alto nivel de liderazgo y compromiso. En cambio, las empresas que tienen a trabajadores con alto nivel de burnout tendrán rotación y bajo nivel de eficiencia.

\section{Burnout}

Como menciona Zhang \& Bartol (2010), al igual que un trabajador puede llegar a tener un nivel muy alto de satisfacción laboral, conocido como engagement, también puede llegar a tener el síndrome de burnout. Davis \& Newstrom (1999) indican que aquel desgaste profesional, cansancio emocional, fatiga laboral es considerado como burnout y que esto atrae al agotamiento, cinismo y baja eficacia laboral. Por este motivo, a las empresas les corresponde que los trabajadores se sientan integrados favorablemente para luego asegurar la retención y así no se sientan despersonalizados de la empresa (De Olivera Borges, Ros-García, \& Tamayo, 2001).

De acuerdo a Reynoso-Nuñez \& Ciófalo-Lagos (2015) en las empresas el agotamiento, despersonalización y baja eficacia laboral son síntomas que perjudican a los empleados y hacen que estos se recluyan y pierdan el encanto de lo que realizan a diario. Es importante que se garantice las condiciones laborales de los individuos al igual que las de la organización, hacer que se sientan identificados, involucrados y atraídos hacia el reto que tienen de conservar su trabajo (Topa \& Moriano, 2012).

Como menciona Maslach \& Jackson (1981), el burnout es analizado como el cambio en la eficacia de un trabajador, el cansancio en su día a día y las pocas energías para el trato diario con las personas a su alrededor.

Grensman, Dev Acharya, Wändell, Nilsson \& Werner (2016), identifican diferentes niveles de gravedad dentro del burnout. Los leves incluyen disconformidades, agotamiento y desgano al ir al trabajo. Los moderados son aquellos que ya evidencian despersonalización, aislamiento y negativismo. Los graves son aquellas personas que realizan su trabajo lentamente o simplemente no asisten a sus funciones.

Es por esta razón como lo indica Cheung \& Tang (2009), dentro de su estudio, que las personas en las organizaciones deben aceptarse como familia y relacionarse para tener una convivencia tranquila, segura y en paz. Las relaciones interpersonales son necesarias para la 
productividad, eficiencia y eficacia de los empleados. Es por esta razón que muchas empresas hoy en día se encuentran implementando proyectos para que sus colaboradores puedan sentirse más comprometidos, más unidos a los ideales de la institución y que no haya cabida en las empresas para colaboradores con el síndrome de burnout. (Chan \& Wyatt, 2007).

\section{Metodología}

\section{Tipo de estudio y unidad de análisis}

El presente trabajo es cuantitativo, exploratorio y descriptivo ya que, según Hernández, Fernández \& Baptista (2014) este tipo de estudios analizan problemáticas poco investigadas y en este contexto y sector, la calidad de vida laboral, el engagement y el burnout son variables que han sido poco estudiadas en Ecuador.

El artículo es de tipo experimental, transeccional donde la característica es recoger datos en un único momento.

Para corroborar si existe o no burnout entre los colaboradores de una IES en Ecuador, se creyó oportuno excluir a los empleados con menos de 2 años en la institución. De tal manera que se cuenta con una población de 290 colaboradores.

\section{Instrumento}

Para medir la calidad de vida laboral, se utilizó el instrumento propuesto por Cabezas (1998) Calidad de Vida Profesional (CVP35). El instrumento contiene 35 ítems, los cuales se evalúan en una escala de Likert, con una puntuación del 1 al 4, en donde 4 representa "mucho" y el 1 representa "nada". Este documento incorpora dimensiones como la motivación intrínseca (10 ítems), Apoyo de Directivos (13 ítems), Carga de trabajo (11 ítems) y un ítem adicional referentes a cómo el trabajador se distingue en su vida profesional (Martín, et al., 2004).

De igual manera, para medir el engagement se utiliza el Work Engagement Scale (UWES-17) propuesto por Shaufeli, Salanova, González-Roma \& Bakker (2002). Éste contiene 17 ítems los cuales se evalúan en una escala de Likert, con una puntación del 0 al 6, en donde el 6 representa "todos los días" y el 0 "ninguna vez". Incorporando las dimensiones de vigor (6 ítems), la dedicación (5 ítems) y la absorción en el trabajo (6 ítems) (Spontón, Medrano, Maffe, Spontón, \& Castellano, 2012).

Para medir el burnout se seleccionó el Maslach Burnout Inventory (MBI-15) de Maslach \& Jackson (1981). Este cuestionario cuenta con 15 ítems, que se evalúan en una escala de Likert con una puntación del 0 al 6, en donde el 6 representa "todos los días" y el 0 "ninguna vez". Los datos recogidos muestran factores como el agotamiento, despersonalización y baja realización personal de los trabajadores (Maslach \& Jackson, MBI: Maslach Burnout Inventory. Manual, 1981). 
Además de estos instrumentos, se recogieron datos demográficos sobre los trabajadores (edad, género, estado civil, nivel académico, antigüedad, horario de trabajo y cantidad de horas laboradas al día).

La compilación de los tres instrumentos contiene un total de 67 preguntas realizadas al personal administrativo y docente de una IES, utilizando en los dos primeros instrumentos una escala de siete (7) posiciones y en la última una escala de cuatro (4) posiciones.

\section{Procedimiento}

El departamento de Recursos Humanos de la IES aprobó la realización del estudio, la aplicación del instrumento se realizó durante el mes de septiembre del 2016 a través de encuestas individuales a los colaboradores. La investigadora fue puesta por puesto explicando el estudio a los trabajadores e indicándoles la confidencialidad de la información. Cada entrevista duró al menos 20 minutos. Todos los materiales físicos fueron archivados en un gabinete. El análisis de los datos se realizó a través de SPSS.

\section{Análisis de los datos}

\section{Demografía}

Tal como se puede observar en la Figura 1, el 57\% de los trabajadores corresponden al género femenino y el $43 \%$ al masculino. En lo que respecta a la edad, el $36 \%$ tienen entre $19 \mathrm{y}$ 29 años, el $25 \%$ entre 30 y 39 años, el $22 \%$ entre 40 y 49 años y el $17 \%$ entre 50 y 65 años. Del total de trabajadores el $53.6 \%$ se encuentran casados, mientras el $46.4 \%$ entre solteros, divorciados, viudo o unión libre.

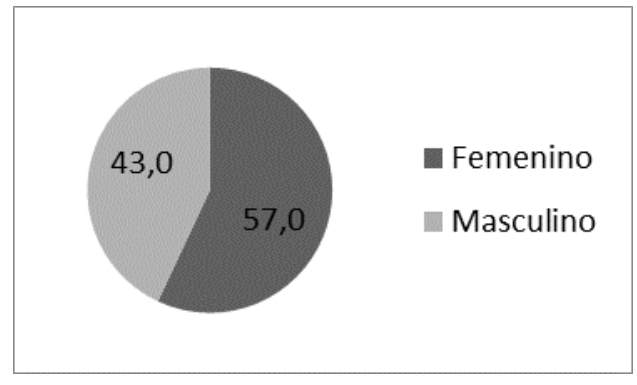

Figura 1 Distribución por Género Elaborado por: Autoras

Tomado de: Encuesta realizada a docentes y administrativos de una IES en Ecuador.

De igual manera, del total de los empleados, el $67.5 \%$ trabajan en el área administrativa y el $32.5 \%$ son docentes de la IES. En lo que respecta a la antigüedad, el $48.7 \%$ tienen laborando en la institución entre 2 a 3 años, el $17.7 \%$ tienen entre 3 a 6 años, el $18.1 \%$ tienen entre 6 a 10 años y el $15.5 \%$ tienen más de 10 años. Referente al horario de trabajo, el 53.2\% indican que trabajan 8 horas diarias, y el $46.8 \%$ trabajan más de 8 horas diarias. (Figura 2) 


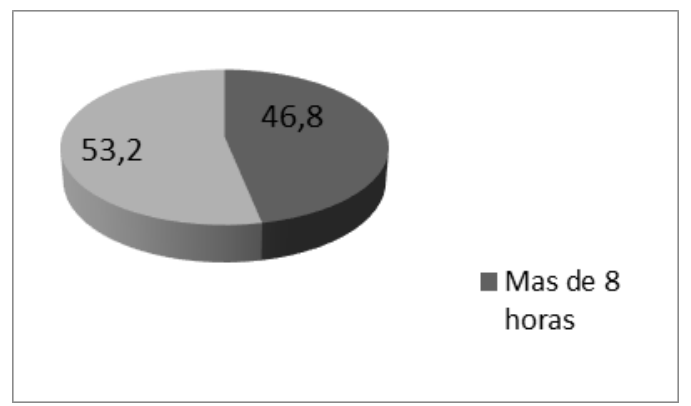

Figura 2 Horas Laboradas

Elaborado por: Autoras

Tomado de: Encuesta realizada a docentes y administrativos de una IES en Ecuador A continuación, se hace un análisis de cada una de los constructos estudiados

\section{Calidad de Vida Laboral (CVP-35)}

Los resultados del CVP-35 fueron: motivación intrínseca con 3.28 sobre 4, percepción de la calidad de vida con 3.09 sobre 4 . Los factores apoyo de directivos y carga de trabajo obtuvieron una media de 2.83 y 2.41 sobre 4 , respectivamente (figura3)

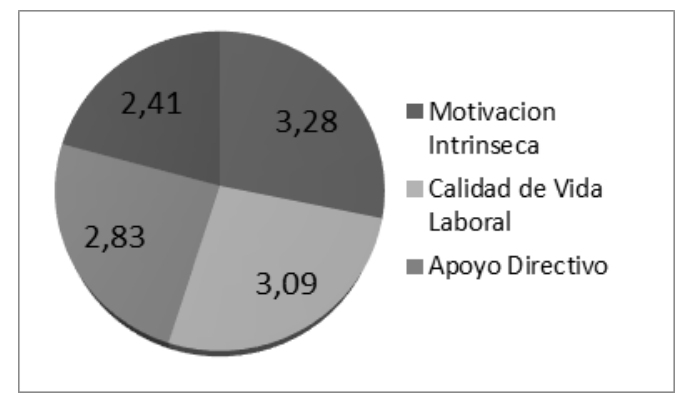

Figura 3 CVP-35

Elaborado por: Autoras

Tomado de: Encuesta realizada a docentes y administrativos de una IES en Ecuador

\section{Engagement}

Los resultados del UWES-17 fueron: vigor y dedicación con un 5.34 sobre 6 y absorción de 4.94 sobre 6. (Figura 4)

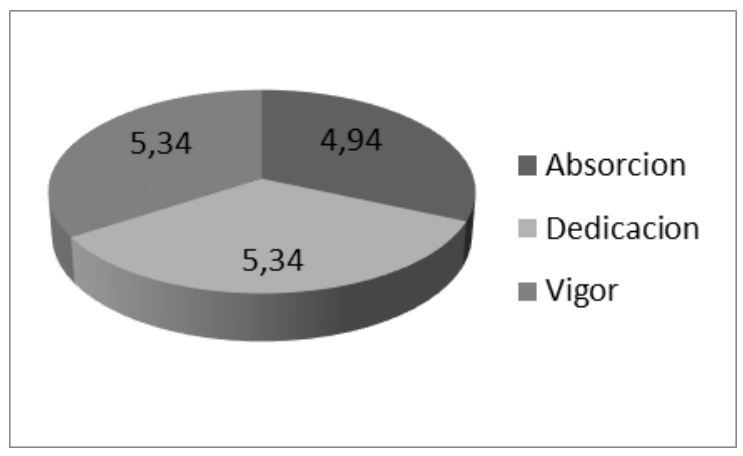


Figura 4

Elaborado por: Autoras

Tomado de: Encuesta realizada a docentes y administrativos de una IES en Ecuador

\section{Burnout}

Por último, en el MBI-15, los resultados fueron en eficacia laboral una media de 5.51 sobre $6 \mathrm{y}$ en agotamiento y despersonalización una media de 2.15 y 1.12 sobre 6 respectivamente (figura $5)$.

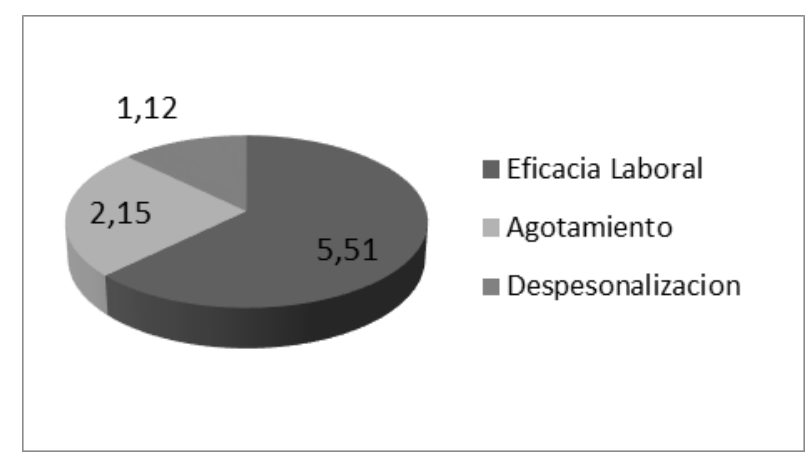

Figura 5 MBI-15

Elaborado por: Autoras

Tomado de: Encuesta realizada a docentes y administrativos de una IES en Ecuador

\section{Conclusiones y recomendaciones}

El objetivo de este artículo fue medir la calidad de vida laboral, el engagement y el burnout en una Institución de Educación Superior del Ecuador. El resultado global muestra que existe una Calidad de Vida Laboral de un 71\%, un $87 \%$ de engagement y un 54\% de burnout dentro de los trabajadores de la IES, tal como se observa en la figura 6.

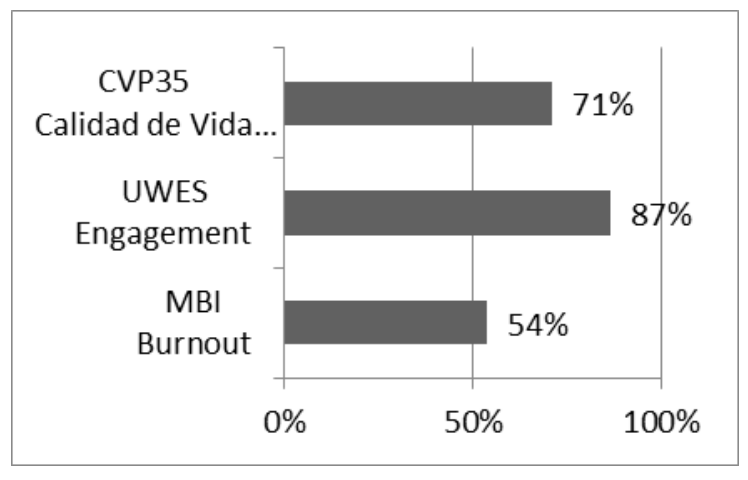

Figura 6 IES

Elaborado por: Autoras

Tomado de: Encuesta realizada a docentes y administrativos de una IES en Ecuador

En relación a los factores que inciden en cada uno de los instrumentos, se toma en cuenta que la IES tiene un alto nivel de motivación intrínseca (CVP35), vigor y dedicación (UWES) y eficacia laboral (MBI). Esto demuestra que los trabajadores de la IES, se encuentran en general complacidos con su organización. 
En la actual IES, se identifica en el instrumento de calidad de vida laboral los ítems que tienen más relevancia como "apoyo de mi familia" y "me siento orgulloso de mi trabajo", de igual manera en engagement lo demuestra el ítem "estoy muy orgulloso de mi trabajo" y "soy muy persistente en mi trabajo". En el caso del instrumento de burnout los ítems de mayor relevancia son "en mi opinión soy bueno en mi puesto" y "contribuyo efectivamente a lo que hace mi organización".

Así mismo, en el caso de los ítems que deben ser estudiados para su mejoría, en el caso de Calidad de vida laboral se encontró “interrupciones molestas", "posibilidad de promoción” y "satisfacción con el sueldo"; en el caso de instrumento de engagement se encontró los ítems de "me es difícil desconectarme de mi trabajo" y "me dejo llevar por mi trabajo"; y por último, en el caso del instrumento de burnout "estoy consumido al final de un día de trabajo" y "estoy emocionalmente agotado por mi trabajo".

Los resultados obtenidos muestran a la IES enfocada a su lema laboral "cuando todos queremos, juntos podemos" dando respaldo y confianza a sus trabajadores. Esta información será base para la implementación de estrategias y nuevas ideas que fortalecerán aún más el engagement ya encontrado a través de los instrumentos.

Se encontró algunas limitaciones en la aplicación de los instrumentos, siendo estas la falta de disponibilidad de los docentes y administrativos y la cantidad de preguntas para contestar. En el caso exclusivo de los administrativos, la limitación fue que no cumplieran con el requisito de tener al menos 2 años en la institución.

De igual manera, la información recopilada en este artículo, es referente para futuras investigaciones en otras IES tanto en el Ecuador como en Sudamérica. De igual manera como futuras investigaciones se sugiere realizar estudios de género y relaciones que determinen la incidencia entre las variables.

\section{Bibliografía}

Adler, N. (2008). International Dimensions of Organizational Behavior. USA: Thomson Learning.

Anónimo. (2002). Beating burn-out. Human Resource Management International Digest, 10(1), 69.

Ardila, R. (2003). Calidad de Vida: Una Definición Integradora. Revista Latinoamericana De Psicología, 35(2), 161-164.

Cabezas, C. (1998). Síndrome de desgate profesional estrés laboral y calidad de vida profesional. FMC, 53-68.

Chan, K., \& Wyatt, T. (2007). Quality of work life: A study of employees in Shangai, China. Asia Pacific Business Review, 501-517. 
Chandranshu, S. (2012). Factors Affecting Quality of Work Life: Empirical Evidence From Indian Organizations. Australian Journal of Business and Management Research, 31-40.

Cheung, F., \& Tang, C. (2009). Quality of work life as a mediator between emotional. Journal of Business and Psychology, 245-255.

Cooper, C. (2009). The transition from the quality of working life to organizational behavior: the first two decades. Journal of Organizational Behavior, 3-8.

Davis, K., \& Newstrom, J. (1999). Comportamiento Humano en el Trabajo. USA: McGraw-Hill.

De Olivera Borges, L., Ros-Garcia, M., \& Tamayo, A. (2001). Socializacion Organizacional: Tacticas y autopercepcion. Revista de Psicologia del Trabajo y de las Organizaciones, 173195.

Esteras, J., Chorot, P., \& Sandin, B. (2014). Predicción del Burnout en los Docentes: Papel de los Factores organizacionales, Personales y Sociodemográficos. Revista de Psicopatología y Psicología Clínica, 79-92.

Grensman, A., Dev Acharya, B., Wändell, P., Nilsson, G., \& Werner, S. (2016). Health-related quality of life in patients with Burnout on sick leave: descriptive and comparative results from a clinical study. International Archives Of Occupational And Environmental Health, 319-329.

Hernandez, R., Fernandez, C., \& Baptista, M. d. (2014). Metodologia de la Investigacion (6ta ed.). Mexico: McGRAW-HILL.

Herrera-Amaya, G., \& Manrique-Abril, F. (2008). Condiciones laborales y grado de satisfacción de profesionales de enfermería. Aquichan, 243-256.

Houdmont, J., Leka, S., \& Bulger, C. (2008). The Definition of Curriculum Areas in Occupational Health Psychology. Occupational Health Psychology, 145-170.

Indumathy, R. (2012). A Study on Quality of Work Life Among Workers with Special Reference to Textile Industry in Tirupur District - A Textile Hub. International Journal of Multidisciplinary Research, 2(4), 265-281.

Jenaro, C., Flores, N., Begoña, M., \& Cruz, M. (2010). Vigour and dedication in nursing professionals: towards a better understanding of work engagement. Journal Of Advanced Nursing, 865-875.

Kahn, W. (1990). Psychological Conditions of Personal Engagement and Disengagement at Work . Academy of Management Journal, 692-724.

Kanfer, R. (1990). Motivation theory and industrial and organizational psychology. Handbook of industrial and organizational psychology, 75-105. 
Martín, J., Cortés, J. A., Morante, M., Caboblanco, M., Garijo, J., \& Rodriguez, A. (2004). Características métricas del Cuestionario de Calidad de Vida Profesional (CVP-35). Gaceta Sanitaria, 129-136.

Maslach, C., \& Jackson, S. (1981). The measurement of experienced burnout. Journal of Occupational Behavior, 99-113.

Maslach, C., \& Jackson, S. E. (1981). MBI: Maslach Burnout Inventory. Manual. USA: MacGraw Hill.

Maslach, C., \& Leiter, M. (1997). The Truth about burnout. San Francisco: Jossey-Bass.

Meyer, J., Becker, T., \& Vandenberghe, C. (1989). Employee motivation and commitment. Journal of Applied Psychology, 991-1007.

Montoya, P., \& Moreno, S. (2012). Relación entre síndrome de burnout, estrategias de afrontamiento y engagement. Psicología desde el Caribe, 205-227.

Nadler, D. A., \& Lawler, E. E. (1983). Quality of Work Life: Perspectives and Directions. Organizational Dynamics, 20-30.

Ocampos, R., Juarez, A., Arias, F., \& Hindrichs, I. (2015). Factores psicosociales asociados a engagement en empleados de un restaurante de Morelos, México. LIBERABIT, 207-219.

Paris, L., \& Omar, A. (2008). Predictores de satisfacción laboral en médicos y enfermeros. Red de Revistas Científicas de América latina, elCaribe, España y Portugal, 233, 244.

Patton, C. (2012). Engagement Means Everyone. Training, 30-31.

Porter, L. W., Bigley, G. A., \& Steers, R. M. (2002). Motivation and Work Behavior. USA: McGraw Hill.

Reynoso-Nuñez, C., \& Ciofalo-Lagos, M. (2015). Socializacion y Engagement. Global Conference on Business and Finance Proceedings, 778-787.

Robbins, M. (2012). Work-related psychological health. Mental Health, Religion \& Culture , 933944.

Schaufeli, W., Salanova, M., Gonzalez-Roma, V., \& Bakker, A. (2002). The Measurement of Engagement and Burnout: A Two Sample Confirmatory Factor Analytic Approach. Journal of Happiness Studies, 71-92.

Segurado, A., \& Agullo, E. (2002). Calidad de Vida Laboral: Hacia un Enfoque Integrador desde la Psicología Social. Psicothema, 828-836. 
Senge, P. (2006). The Fifth Discipline: The Art \& Practice of The Learning Organization. USA: Doubleday.

Solomon, M. (2010). Employee Engagement: The Key to Improving Performance. International Journal of Business and Management, 89-96.

Spontón, C., Medrano, L. A., Maffe, L., Spontón, M., \& Castellano, E. (2012). Validación del Cuestionario de Engagement UWES a la Población de Trabajadores de Córdoba, Argentina. Liberabit. Revista de Psicología, 147-154.

Suescùn-Carrero, S., Sarmiento, G., Alvarez, L., \& Lugo, M. (2016). Calidad de vida laboral en trabajadores de una Empresa Social del Estado de Tunja, Colombia. Rev Med Risaralda, 14-17.

Topa, G., \& Moriano, J. (2012). Tácticas de socialización, satisfacción laboral y abandono entre los soldados españoles: El papel mediador del aprendizaje de los valores organizacionales. Revista Mexicana de Psicologia, 57-68.

VanScotter, J., \& Motowidlo, S. (1997). Interpersonal Facilitation and Job Dedication as Separate Facets of Contextual Performance. Journal of Applied Psychology, 525-531.

Zhang, X., \& Bartol, K. (2010). Linking Empowering Leadership and Employee Creativity: The Influence of Psychological Empowerment, Intrinsic Motivation, and Creative Process Engagement. Academy of Management Journal, 107-128.

Zia, M., Rezvani, M., \& Fattaneh, S. (2014). Investigating the Effect of Emotional Intelligence Components on Organizational Maturity and Job Burn-Out. International Review of Management and Business Research, Vol.3. 